\title{
Indicadores de deserción escolar y su posible atención a nivel medio superior: un caso de estudio
}

\section{Proposal for follow-up to practical activities in a higher-level presential course}

SÁNCHEZ-LÓPEZ, Guillermina†*, JIMÉNEZ-JIMÉNEZ, Yazmín y MORENO-AGUILAR, Ma. Antonia

Benemérita Universidad Autónoma de Puebla, Instituto Tecnológico de Puebla. 4 Sur \#104; Col. Centro C.P. 72000; Puebla de Zaragoza, Puebla, México.

ID $1^{\text {er }}$ Autor: Guillermina, Sánchez-López / ORC ID: 0000-0001-5866-9362, Researcher ID Thomson: S-6946-2018, CVU CONACYT ID: 626579

ID $1^{\text {er }}$ Coautor: Yazmín, Jiménez-Jiménez, / ORC ID: 0000-0002-5065-0162, Researcher ID Thomson: X-3354-2019, CVU CONACYT ID: 552316

ID $2^{\text {do }}$ Coautor: Ma. Antonia, Moreno-Aguilar / ORC ID: 0000-0003-3587-9302, Researcher ID Thomson: S-6713-2018, CVU CONACYT ID: 467317

DOI: $10.35429 / J P D .2019 .7 .3 .19 .29$ Recibido 5 de Enero, 2019; Aceptado 20 de Marzo, 2019

\section{Resumen}

Actualmente es necesario que los egresados del nivel medio superior desarrollen destrezas, habilidades actitudes y aptitudes que les permitan desenvolverse en la siguiente etapa ya sea laboral o académica a la cual se inserten, una vez concluido su preparatoria. A nivel administrativo en toda institución educativa se tienen estándares mínimos a cumplir y que son evaluados por las certificadoras externas, según corresponden a cada centro educativo. Estos indicadores son motivo de acciones punitivas ejercidas contra la institución en sí, pero más allá de estas situaciones hay una preocupación más relevante para las autoras del presente trabajo: la deserción escolar, ya que como es sabido según el reglamento de cada institución cada estudiante tiene 3 o 4 oportunidades de aprobar los cursos, pasados los cuales el estudiante es dado de baja definitivamente del centro educativo. Al interior de las instituciones ¿será posible implementar estrategias que permitan combatir la deserción escolar?.

Evaluación, Deserción, Indicadores Proyectos

\begin{abstract}
Currently it is necessary for graduates of the upper middle level to develop skills, abilities, attitudes and aptitudes that allow them to perform in the next stage, whether work or academic, to which they are inserted, once their high school is finished. At the administrative level in every educational institution there are minimum standards to be met and they are evaluated by external certifiers, as they correspond to each educational center. These indicators are grounds for punitive actions against the institution itself, but beyond these situations there is a more relevant concern for the authors of the present work: school dropout, since as is known according to the regulations of each institution each student has 3 or 4 opportunities to pass the courses, after which the student is permanently withdrawn from the educational center. Within the institutions, will it be possible to implement strategies to combat school dropout?.
\end{abstract}

Evaluation, Attrition, Indicators Projects

Citación: SÁNCHEZ-LÓPEZ, Guillermina, JIMÉNEZ-JIMÉNEZ, Yazmín y MORENO-AGUILAR, Ma. Antonia. Indicadores de deserción escolar y su posible atención a nivel medio superior: un caso de estudio. Revista de Didáctica Práctica. 2019. 3-7: 19-29.

\footnotetext{
*Correspondencia al Autor (Correo Electrónico: guille.sanlopez@gmail.com)

$\dagger$ Investigador contribuyendo como primer autor.
} 


\section{Introducción}

En el plan educativo Nacional se menciona que la educación media superior se hizo obligatoria a partir del 2012, para 2017, se sabía que el $40 \%$ de los egresados del NMS, no alcanzaban las capacidades necesarias para insertarse al terreno laboral, como lo mencionó, el entonces subsecretario de Educación Media Superior, Rodolfo Tuirán Gutiérrez. Los resultados de las pruebas estandarizadas. PISA, EXCALE Y ENLACE, nos dan muestra de la realidad que presentan los estudiantes inscritos a este nivel educativo. Lo anterior representan puntos de alerta que las instituciones educativas tienen la responsabilidad de atender, corregir y mejorar, considerando como Institución a docentes, directivos, estudiantes y padres de familia.

En los últimos años, numerosos cambios se han realizado con la finalidad de mejorar la calidad en el nivel medio superior, se incrementó el número de: planteles, profesores, alumnos atendidos, pero las "bases" bajo las cuales se desarrollan los planes de estudio, no fueron modificadas.

Como lo plantea Schmelkes, (2001), en la educación, dependiendo del alcance de la misma se tienen objetivos tanto externos como internos, el objetivo externo primordial es contribuir al mejoramiento de la calidad de vida -actual y futura - de los educandos, y de esta manera a la calidad de los procesos de desarrollo de la sociedad.

Es importante mencionar que de la labor realizada al interior de las instituciones educativas depende, que los alumnos asistan a la escuela, lleguen puntuales, cuenten con lo necesario para poder aprender, reciban el apoyo extraescolar indispensable para el adecuado logro de los objetivos educativos.

En las escuelas es donde se desarrolla la formación del individuo que participará de manera activa en otro plantel escolar del siguiente nivel educativo, o, en el mercado laboral, en general en la sociedad.

De manera tal que como dice Schmelkes, (2001), puede decirse que la que se beneficia por la labor de un centro educativo, es la comunidad donde el egresado se desempeñará profesional, social y políticamente.
Por lo que es necesario que las instituciones como tal visualicen al realizar todas sus planeaciones que los resultados cualitativos del quehacer docente se reflejarán, no solo cuando cada alumno está dentro de sus aulas, sino que su labor trasciende en el tiempo. El mejoramiento de la calidad de toda institución, sea pública o privada, educativa o empresarial, se caracteriza por poner como centro al que está siendo beneficiado por su labor. En el caso de las instituciones educativas, el foco de atención para el mejoramiento de la calidad debe estar puesto en:

- El estudiante de hoy.

- Ese mismo estudiante mañana.

- Los padres de familia.

- La institución que lo recibe como egresado.

- La persona u organización que le da empleo.

- La comunidad en la que el alumno vive.

- La sociedad en la que se desarrollará social, económica, cultural y políticamente.

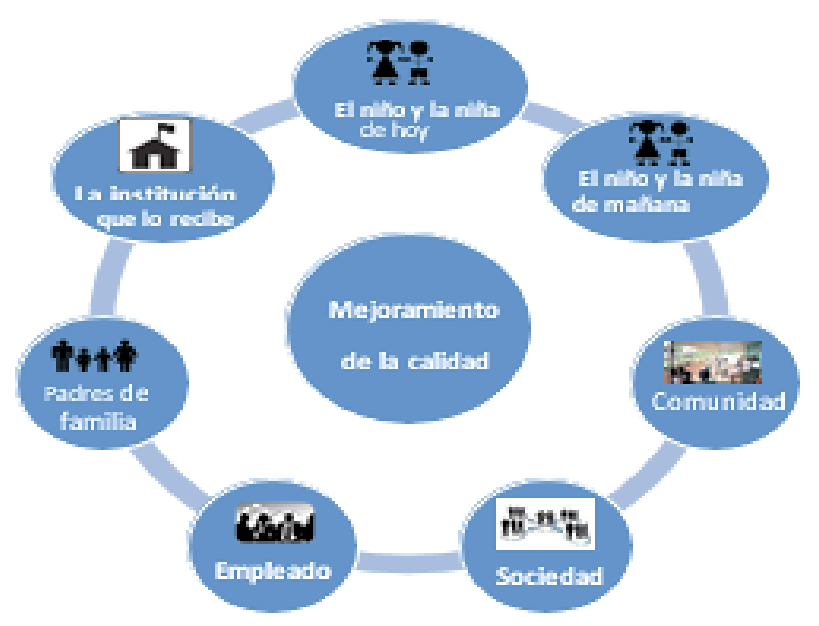

Figura 1 ¿Para quién mejorar la calidad?

De la ilustración 1 se observa que la mejora de la calidad educativa, debe entenderse como la necesidad de satisfacer a todos sus usuarios o beneficiarios, tanto los externos como los internos, ya que son estos los que evaluarán el trabajo realizado por la institución, es decir, alumnos, egresados, padres de familia, empleadores, comunidad y sociedad. 
Es por ello imperioso conocer: necesidades, expectativas, preocupaciones, insatisfacciones, con respecto al servicio que la institución brinda. Recordando que la calidad es un movimiento cuya trayectoria es una espiral ascendente, una vez iniciado, lo único que debe lograr es... más calidad. (Schmelkes, 2001).

Es importante para toda institución determinar las necesidades de los estudiantes, si bien es prioridad la apropiación de conocimientos y el cumplimiento de los objetivos de aprendizaje, no puede dejarse a un lado la formación de valores, las necesidades de los estudiantes o la actividad deportiva, para saber que priorizar, que incorporar, y como proceder para lograr ese aprendizaje significativo, es decir, lograr una calidad total. Según Schmelkes, (2001), la calidad en las instituciones de nivel medio superior, se ve afectada por:

- La no inscripción.

- La deserción.

- La reprobación.

- El problema del no aprendizaje.

- El ambiente.

- La indisciplina.

- El tiempo real de clase.

- Los recursos para la enseñanza.

- Las relaciones con la comunidad.

- Las relaciones entre el personal de la escuela.

Un proceso de mejora continua, con la finalidad de lograr y mantener la calidad debe identificar el problema, establecer las causas y los efectos que provocan y plantear una estrategia que ataque el problema de raíz. $\mathrm{La}$ Secretaria de Educación Pública, SEP, en Puebla reportó que en el ciclo escolar 20172018, las principales causas de abandono escolar, detectadas, fueron: factores personales, económicos, cambios de institución, violencia o acoso institucional. falta de interés en las clases.
Estrada, (2014), indica que, en diversas Investigaciones Educativas, se observa que en la etapa de los 13 hasta los 18 años, los adolescentes pasan por diversas situaciones que al entrelazarse entre sí tienen consecuencias que desatan el abandono escolar.

Weis, (2014), nos muestra que el costo del bachillerato puede ser muy caro, al incluir pasajes, materiales, comida, imprevistos de salud, abandono por el padre o la madre, lo cual aunado a cambio de residencia o a "malas compañías", da como resultado la "necesidad" de dejar los estudios.

Diversos autores coinciden en que la mayor de las causas de abandono escolar es la reprobación y por tanto la auto descalificación que el estudiante empieza a desarrollar, generando un efecto desmotivante hacia el proceso de aprendizaje. (Merino, 1993; Piña, 1997; Espíndola y León, 2002; Orozco, 2004).

Esta desmotivación provoca que a pesar de que la institución cuente con procesos de recuperación de las diversas asignaturas, los alumnos simplemente no se presentan o bien realizan entregas incompletas de manera que reprueban nuevamente y al sentirse desafiliados, ajenos e incompetentes simplemente abandonan la institución educativa. (Weis, 2014).

Los gobiernos federales crean diversas "estrategias" para apoyar la continuidad de los estudios en los alumnos del nivel medio superior, mismas que no arreglan completamente la situación, pero si contribuyen a mitigar un poco las diversas problemáticas por las cuales atraviesan. En nuestro caso, la preparatoria regional "Simón Bolívar", tiene dentro de sus principales lineamientos llevar a cabo procesos de mejora continua tanto administrativa como académicamente. Para el caso de este proyecto se tomó en cuenta la deserción elevada que se produjo como resultado de la implementación del primer semestre del plan 07 , una situación por demás grave y preocupante, que nos hizo reflexionar si las estrategias de enseñanza aprendizaje planteadas al interior de las academias eran adecuadas o no, y por otra parte, si las evidencias solicitadas, realmente apoyaron a la apropiación de los conocimientos de los estudiantes o no. 


\section{Problemática}

En el caso de la preparatoria Regional "Simón Bolívar" (PRSB), al término del primer semestre del plan 07, de los 8 grupos de primero matutino, se tuvieron 46 estudiantes de un total aproximado de 320 para examen extraordinario, de la materia de Algebra, en contraste con una minoría para examen de informática y para química.

El reglamento de ingreso, permanencia y egreso del nivel medio superior de la BUAP, establece que, si un estudiante reprueba el extraordinario II, será dado de baja. Siendo lo anterior un reto para la institución en cuestión, derivado de lo cual se plantean las siguientes preguntas de investigación.

¿Qué factores provocan la alta reprobación de los estudiantes de las asignaturas mencionadas?

¿Cómo lograr que el índice de deserción disminuya?

¿Cómo lograr un aprendizaje
significativo?

¿Qué acciones pueden implementarse al interior de las academias para mejorar esta condición?

\section{Objetivo}

1. Determinar las causas que provocan la reprobación en los estudiantes de la PRSB.

2. Plantear una estrategia de mejora continua académica dentro de la institución.

\section{Objetivo específico:}

1. Plantear una estrategia de mejora continua en la materia de algebra.

\section{Metodología}

Para contestar la primera pregunta de investigación se realizó una encuesta a los alumnos de primeros años tanto matutino, como vespertino, la muestra fue de 15 alumnos: 8 mujeres y 7 hombres de cada grupo, esto debido a que en la institución hay un mayor número de alumnas.
La encuesta aplicada contiene las siguientes preguntas:

¿Cuántas materias reprobaste en el primer semestre? semestre?

¿Qué materias reprobaste en primer

¿Qué evidencias te faltaron para aprobar la (s) materia (S)?

¿Conocías de manera personal a algunos de los alumnos que se dieron de baja?

¿Qué causas consideras que provocaron su reprobación?

¿Cuál consideras que fue la materia más difícil durante el primer semestre?

¿Cuál consideras que fue la materia más difícil durante el primer semestre?

¿Planeas continuar con tus estudios?

¿Tu panorama personal, familiar y económico te permite concluir con tu educación media superior?

\section{Tipo de Investigación}

La presente investigación se realiza bajo un enfoque cuantitativo, mediante un proceso deductivo que plantea inicialmente analizar las causas del alto índice de reprobación en la preparatoria regional "Simón Bolívar" de la Benemérita Universidad Autónoma de Puebla, utilizando diversos instrumentos de evaluación validados. Las Fuentes utilizadas para el proyecto tienen como base las siguientes fases:

Una Encuesta realizada a una muestra de 410 estudiantes de la mencionada institución tanto del turno matutino como del vespertino teniendo por tanto una confiabilidad del $98 \%$ en nuestros resultados.

institucionales. 


\section{Resultados de la aplicación de la encuesta}

Tras el estudio estadístico se obtuvo para el turno matutino, que el $38 \%$ de los encuestados indicaron como se muestra en el Gráfico 1, que algebra es la materia más difícil, seguida muy cercanamente por química con un $36 \%$.

Materias más difíciles 1er semestre

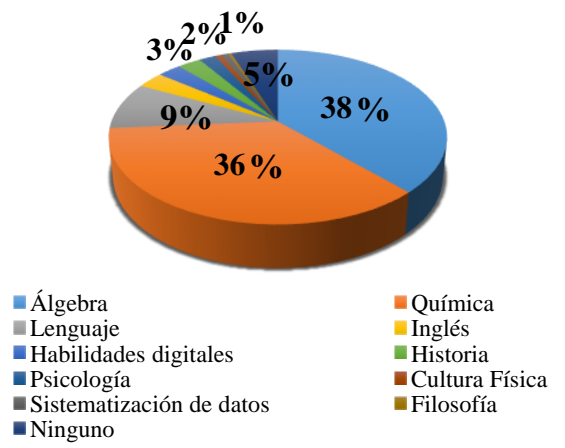

Gráfico 1 Turno matutino "materias más difíciles" Fuente: elaboración propia

El $49 \%$ de los encuestados del turno vespertino, indicaron como lo muestra el gráfico 2, que la materia más difícil de primer semestre fue Química, seguida por algebra con un $17 \%$.

Materias más dificiles en segundo semestre

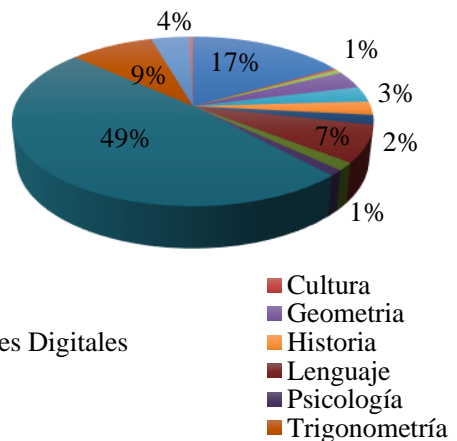

Gráfico 2 "Materias más difíciles" turno vespertino" Fuente: elaboración propia

Al preguntarles el porqué de su respuesta, indicaron como causa principal que los temas son complicados y como segunda causa está la forma de enseñanza que provoca desinterés. Gráfico 3.

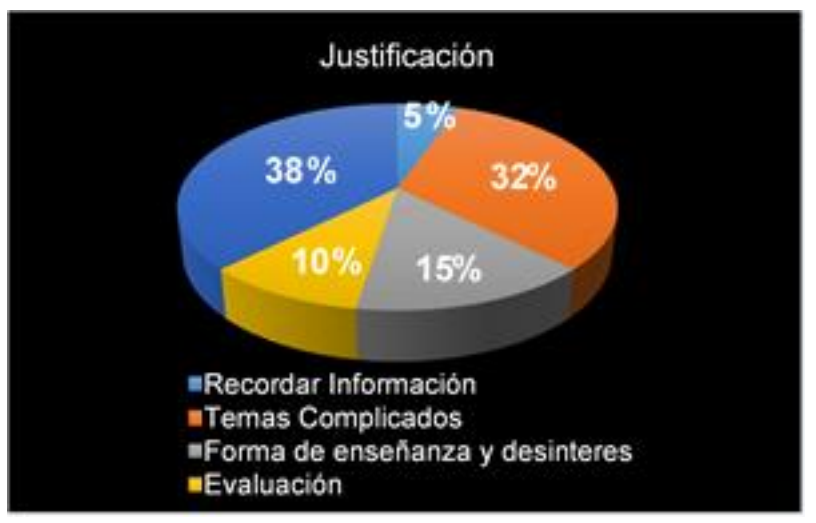

Gráfico 3 ¿Por qué reprobaste?

Fuente: elaboración propia

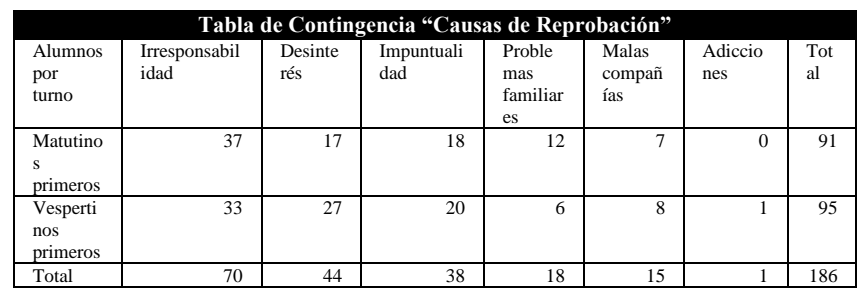

Tabla 1 Tabla de contingencia

\section{Causas de reprobación}

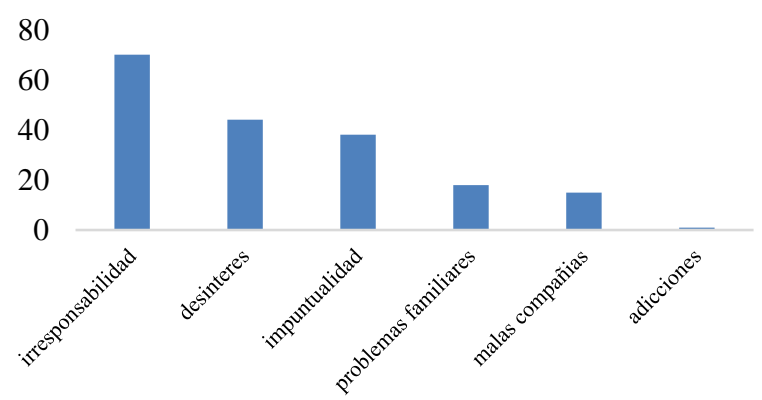

Gráfico 1 Causas de reprobación

Fuente: elaboración propia

Que la mayoría de los encuestados indicó que reprobó materias por su irresponsabilidad y desinterés en el proceso educativo.

De lo anteriormente planteado se presenta el siguiente diagrama de proceso donde se muestran todas las variables en estudio a considerar. 


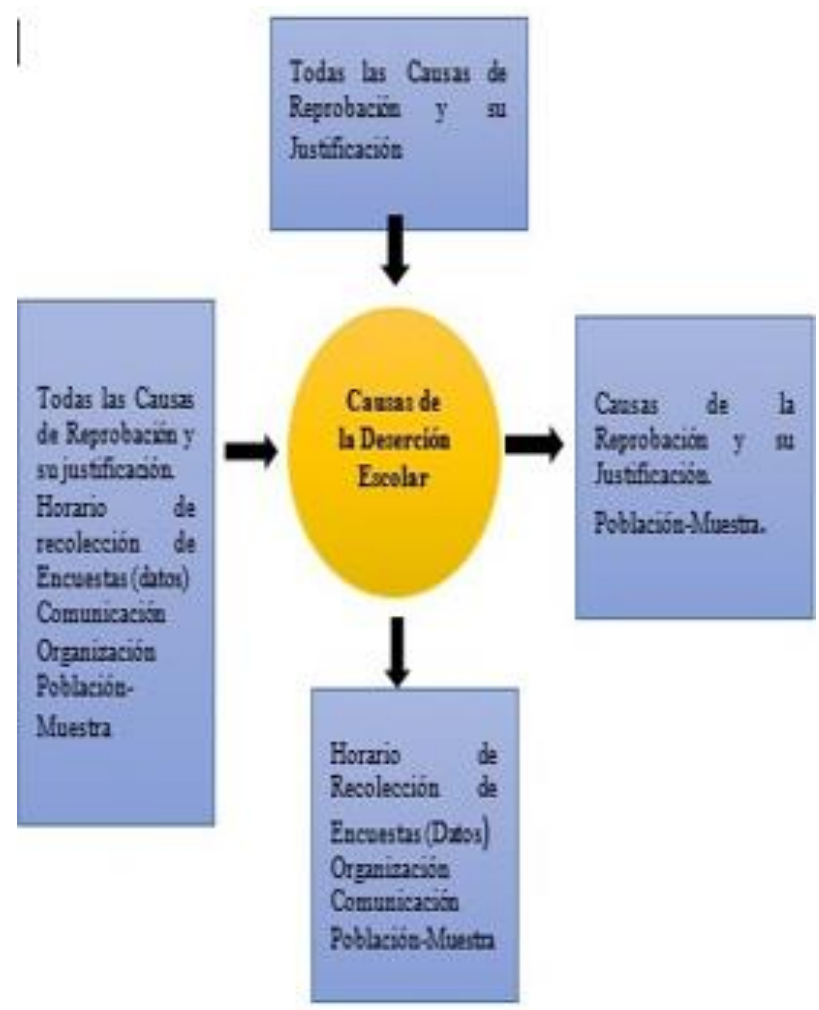

Figura 1 Diagrama de Proceso 1 Causas de la deserción escolar

Fuente: elaboración propia

Como podemos observar las causas de reprobación son variadas y no solo son imputables al docente frente a grupo sino a una serie de variables presentes en todo proceso educativo, las cuales se aprecian en el diagrama de Ishikawa siguiente.

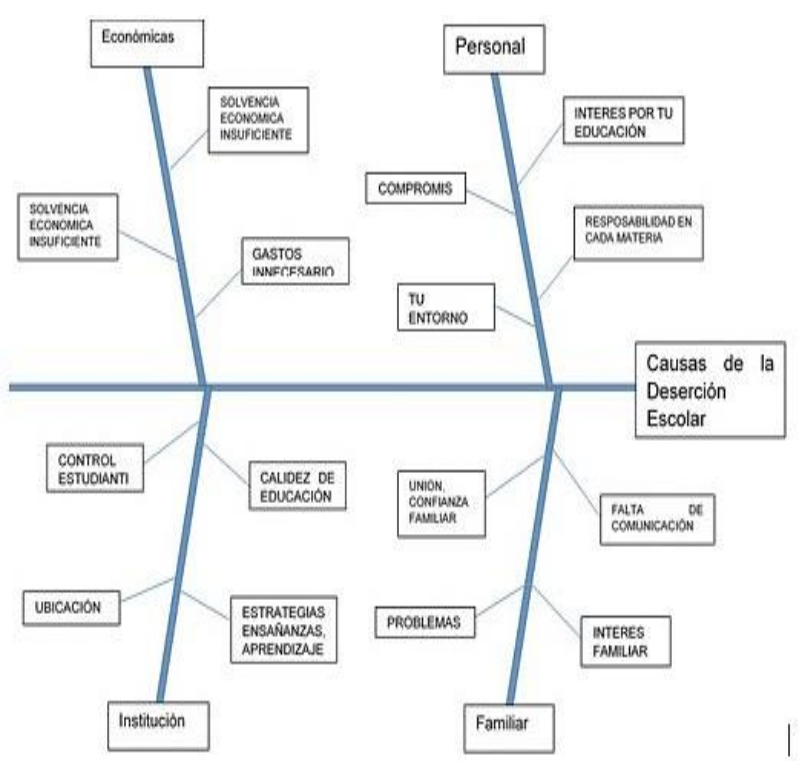

Figura 2 Diagrama de Proceso 2 Ishikawa

Fuente: elaboración propia

Como resultado de la investigación realizada se establece la siguiente propuesta de mejora continua a implementar en la Preparatoria Regional "Simón Bolívar" de la Benemérita Universidad Autónoma de Puebla.
1.- Considerando que la causa de reprobación que más se repitió fue la Irresponsabilidad, se solicita a las integrantes del APOEd, (Apoyo psicológico y de orientación educativa), así como a los tutores de grupo, la implementación de actividades de concientización en los estudiantes de modo que pueda mejorarse esta causa.

2.- Con respecto a la segunda causa de reprobación: Desinterés, la Academia de unidad de matemáticas propone la implementación de una serie de actividades que promuevan la motivación en los estudiantes, entre ellas están la creación del laboratorio de matemáticas, donde el estudiante pueda ver de una manera más practica los conceptos teóricos, estudiados en clase, este tipo de actividades utilizarán la socialización como detonante para el aprendizaje, para que como lo plante Vygotsky, la zona de desarrollo proximal, pueda ser desarrollada por ellos.

3. Otro punto que los autores del presente trabajo detectaron fue la necesidad de aprovechar las actividades lúdicas para involucrar a los estudiantes con el aprendizaje de las matemáticas como lo plantean Ceferino \&Guadalupe, (2007)

4.- Al término de la primera implementación se propone realizar un análisis de resultados, estableciendo el grado de deserción escolar obtenida, con la finalidad de valorar la viabilidad de seguir llevando a cabo el laboratorio de matemáticas.

\section{Implementación de la propuesta}

Es importante resaltar que esta propuesta empezó a ponerse en práctica en el semestre enero-junio de 2019. Las prácticas de "Laboratorio de Matemáticas" que se proponen constan en su formato de:

- Introducción.

- Objetivos.

- Desarrollo experimental.

- Resultados: mediciones, análisis.

- Conclusiones. 
En la siguiente parte se detalla una de las 4 prácticas que se llevaron a cabo, las características de estas es que permitían aterrizar y poner a la práctica los conocimientos adquiridos en el proceso aprendizajeenseñanza.

Ejemplo:

Práctica 2 de Geometría: "Construcción de una calculadora para resolución de triángulos oblicuángulos"

Docente:

Alumno:

Grupo:

Introducción:

Un triángulo es oblicuángulo cuando sus tres lados son oblicuos, es decir, no tienen ángulo recto $\left(90^{\circ}\right)$. Este tipo de triángulo se resuelve mediante la ley de seno y cosenos.

Ley de senos:

La razón que existe entre un lado de un triángulo oblicuángulo y el seno del ángulo opuesto a dicho lado es proporcional a la misma razón entre los lados y ángulos restantes.

Simbólicamente se tiene:

Ley de senos

$\frac{a}{\operatorname{Sen} A}=\frac{b}{\operatorname{Sen} B}=\frac{c}{\operatorname{Sen} C}$

También como:

$\frac{\operatorname{Sen} A}{a}=\frac{\operatorname{Sen} B}{b}=\frac{\operatorname{Sen} C}{c}$

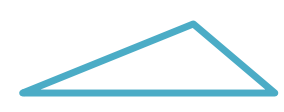

La ley de senos es utiliza cuando:

* Los datos conocidos son 2 lados y el ángulo opuesto a uno de ellos.

* Los datos conocidos son dos ángulos y cualquier lado.

Ley de cosenos.

El cuadrado de un lado de un triángulo oblicuángulo es igual a la suma de los cuadrados de los lados restantes, menos el doble producto de dichos lados por el coseno del ángulo opuesto al lado buscado.

Expresado matemáticamente:
Ley de cosenos:

$a^{2}=b^{2}+c^{2}-2 b c \operatorname{Cos} A$
$b^{2}=a^{2}+c^{2}-2 a c \operatorname{Cos} B$
$c^{2}=a^{2}+b^{2}-2 a b \operatorname{Cos} C$

La ley de cosenos se utiliza cuando:

* Se tiene el valor de dos lados y al ángulo comprendido entre ellos.

* Se tiene el valor de los tres lados.

El procedimiento para calcular los lados y ángulos de los triángulos oblicuángulos es de manera aritmética, sin embargo, se puede construir una calculadora en Excel que permita obtener los datos de lados y ángulos faltantes solo con poner los datos que necesarios para resolver este tipo de triángulos.

La construcción de esta calculadora no es complicada pues solo es necesario conocer el funcionamiento de las operaciones básicas en Excel utilizando solamente las celdas. Sin embargo también se puede utilizar Microsoft VBA (Visual Basic for Applications) que es un lenguaje de macros que se emplea para crear aplicaciones que permiten ampliar la funcionalidad de programas de la suite Microsoft Office.

Se puede señalar que Visual Basic para Aplicaciones es un subconjunto casi completo de Visual Basic, Microsoft VBA al estar incluido dentro del Microsoft Office, puede emplearse tanto en Word, Excel, Access así como en Powerpoint.

La principal utilidad radica en poder automatizar tareas frecuentes o cotidianas.

Objetivos: Aprender programar la ley de seno y cosenos para resolver cualquier triángulo oblicuángulo.

- Uso básico de Excel.

- Aplicar conocimiento de trigonometría.

Planteamiento del problema.

Como continuación se desea dar solución al siguiente problema (problema que se abordara en la siguiente práctica): 
En el periodo inter ciclo escolar, se realizarán trabajos de mantenimiento en la Preparatoria Regional "Simón Bolívar", para lo cual el maestro Armando Torres, secretario administrativo, debe realizar la petición del trabajo ante las instancias correspondientes de la BUAP, uno de los requisitos que le piden es el cálculo del área de la fachada del edificio que habrán de pintar, con la finalidad de determinar la cantidad de pintura que debe comprarse, así como el número de trabajadores que deberán asistir.

¿Puedes ayudar al maestro Armando a determinar el área de cada pared del edificio SB2 de la preparatoria?

Para dar soluciona al problema anterior se realizará la construcción de esta calculadora con el objetivo de que los alumnos tengan una herramienta que les permita realizar sus cálculos de manare más fácil y rápida.

\section{Materiales:}

- Computadora que tenga la paquetería de office.

- Libreta.

- Lápiz.

\section{Procedimiento.}

1. En una hoja de cálculo definir los datos que se tienen de la práctica anterior. (Se realiza el ejemplo para ley de senos cuando los datos conocidos son 2 lados y el ángulo opuesto a uno de ellos).

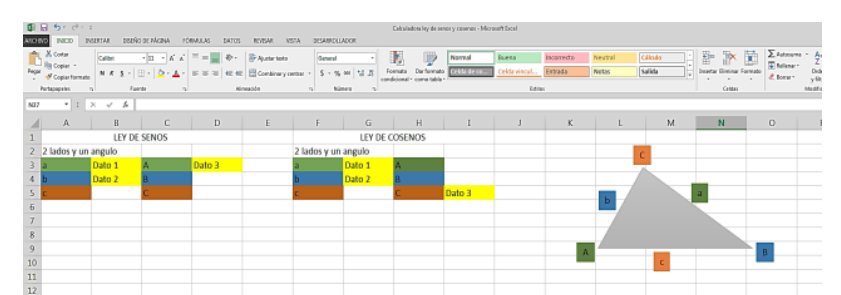

Calculadora Ley de Senos y Cosenos

Figura 3 Definición de datos

2. Para calcular el ángulo $B$ utilizamos los datos conocidos (Dato1=a, Dato2=b, Dato3=A) y la ley de senos utilizando la relación siguiente:

$\frac{a}{\operatorname{Sen} A}=\frac{b}{\operatorname{Sen} B}$

\section{obtener B}

Realiza el despeje correspondiente para

$\mathrm{B}=$

3. El resultado del despeje anterior, se captura en Excel utilizando las celdas de cada dato que se tiene (Dato1=a, Dato2=b, Dato3=A) Figura 4 (Se utiliza la función $=$ GRADOS para convertir el resultado a grados, la función =ASENO es para definir el seno inverso y la función =RADIANES es para convertir los grados a radianes y poder utilizar las razones trigonométricas de Excel.

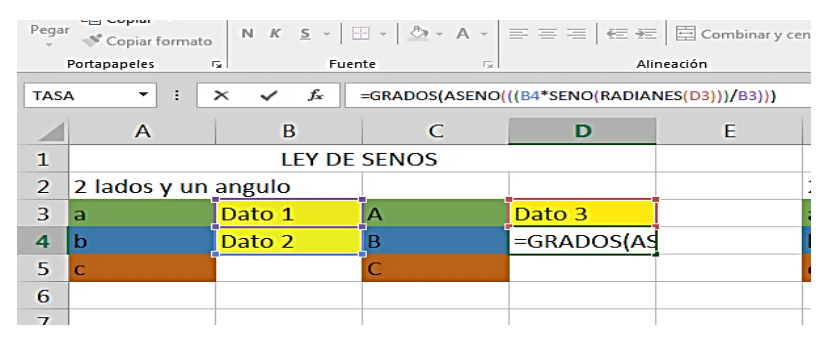

Figura 4 Captura de datos

4. Al obtener el ángulo $\mathrm{B}$, de manera fácil podemos obtener el ángulo $\mathrm{C}$, puesto que en Excel solo es realizar la siguiente operación con celdas.

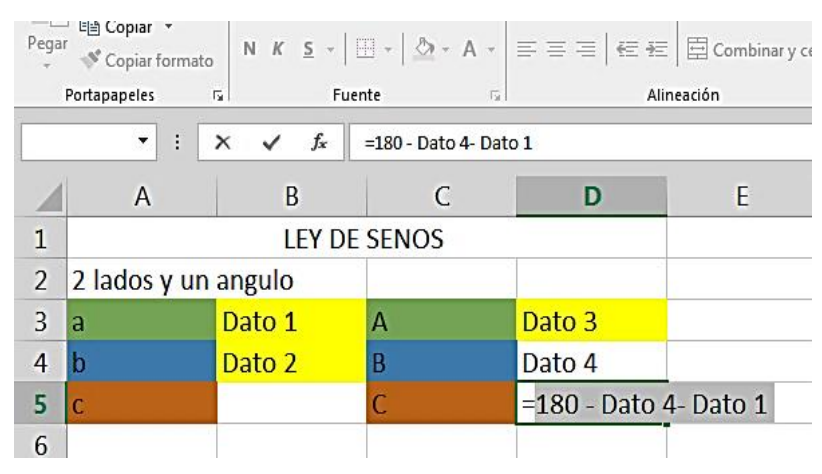

Figura 5 Obtención de la hipotenusa

5. Para calcular el lado c podemos ocupar la siguiente relación

$$
\frac{b}{\operatorname{Sen} B}=\frac{c}{\operatorname{Sen} C}
$$

Realiza el despeje de c

$c=$

6. Del despeje anterior, captura en Excel utilizando las celdas de cada dato que se tiene $($ Dato2=b, Dato4=B, Dato5=c), Figura 6 : 


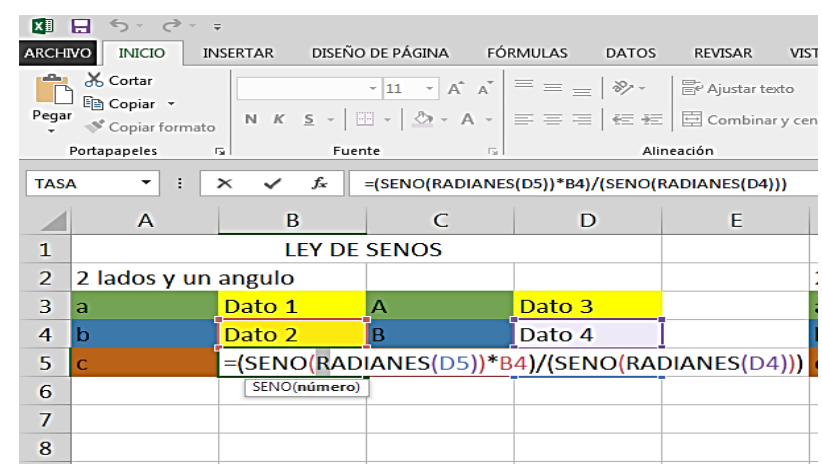

Figura 6 Captura de datos

7. Verifica con un ejercicio resuelto que cumpla con las especificaciones de este ejercicio para verificar que tu programa funcione de manera correcta.

Para ley de cosenos se construye de manera parecida a la ley de senos.

1. En una hoja de cálculo definir los datos que se tienen de la práctica anterior. (Se realiza el ejemplo ley de cosenos para cuando se tiene el valor de dos lados y al ángulo comprendido entre ellos).

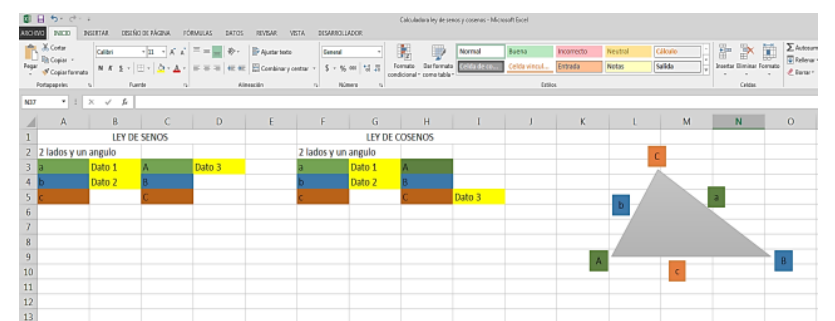

\section{Calculadora Ley de Senos y Cosenos}

Figura 7 Captura de datos para ley de cosenos

2. Para calcular el lado c utilizamos los datos conocidos (Dato1=a, Dato2=b, Dato3=A) y la ley de cosenos utilizando la relación siguiente:

$$
c^{2}=a^{2}+b^{2}-2 a b \operatorname{Cos} C
$$
obtener c

Realiza el despeje correspondiente para

$$
\mathrm{C}=
$$

3. El resultado del despeje anterior, se captura en Excel utilizando las celdas de cada dato que se tiene (Dato1=a, Dato2=b, Dato3=C) ver la figura siguiente:
(Se utiliza la función =RADIANES es para convertir los grados a radianes y poder utilizar las razones trigonométricas de Excel).

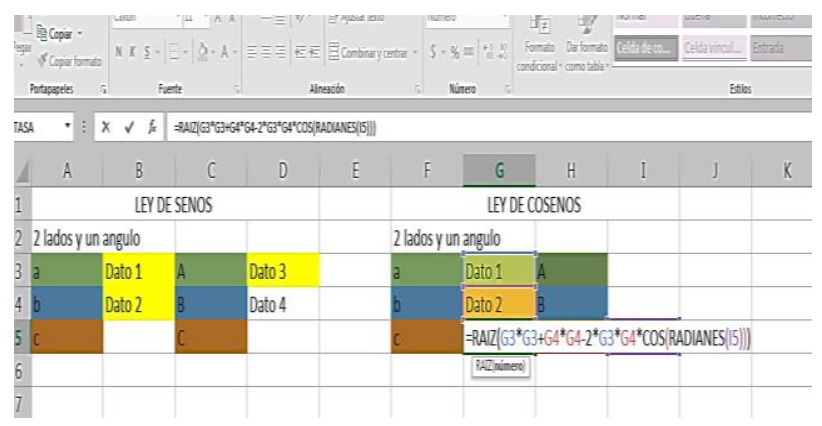

Figura 8 Ley de cosenos

4. Para calcular el ángulo B podemos utilizar ley de senos o cosenos con la siguiente relación:

$$
b^{2}=a^{2}+c^{2}-2 a c \operatorname{Cos} B
$$

Despeje a B

$\mathrm{B}=$

5. Del despeje anterior captura en Excel utilizando las celdas de cada dato que se tiene (Dato1=a, Dato2=b, Dato4=c) ver Figura 9:

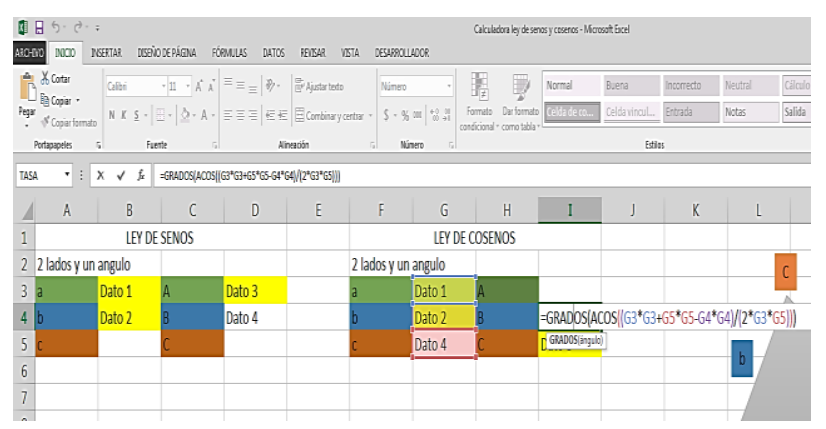

Figura 9 Ley cosenos captura

8. $\quad \mathrm{Al}$ obtener el ángulo $\mathrm{B}$, de manera fácil podemos obtener el ángulo $\mathrm{A}$, puesto que en Excel solo es realizar la siguiente operación con celdas.

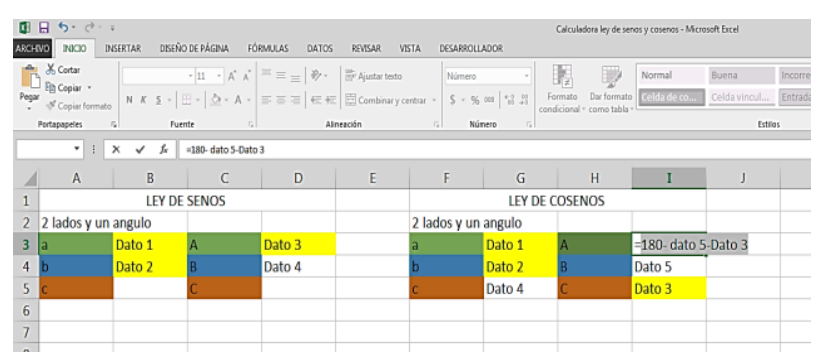

Figura 10 Operaciones para Ley de cosenos 
6. Verifica con ejercicio resuelto que cumpla con las especificaciones de este ejercicio para verificar que tu programa funcione de manera correcta.

En esta práctica se trabajó con la ley de seno y cosenos con las siguientes especificaciones:

La ley de senos.

* Los datos conocidos son 2 lados y el ángulo opuesto a uno de ellos.

La ley de cosenos.

Se tiene el valor de dos lados y al ángulo comprendido entre ellos

Se deja como actividad para los alumnos el programar la ley de senos tomando en cuenta que los datos conocidos son dos ángulos y cualquier lado, mientras que, para la ley de cosenos se tiene el valor de los tres lados.

\section{Conclusiones}

La motivación del estudiante es una labor que debe desarrollarse como parte del curriculum oculto de las diferentes asignaturas. En el caso de matemáticas también es importante tomarla en cuenta, ya que hay una predisposición de los estudiantes hacia "pensar" que la asignatura es "difícil" y que irremediablemente reprobarán, lo cual ya en sí es el primer impedimento al cual se enfrentan los docentes de cada institución. Cabe hacer mención que la presente propuesta fue aplicada en su totalidad en 7 grupos de primer año de preparatoria y de manera parcial en uno más, cada grupo con cuarenta estudiantes aproximadamente. Tras la aplicación de las prácticas del Laboratorio de Matemáticas, se obtuvieron los siguientes resultados:

- Se observó la asistencia más participativa por parte de los estudiantes, ya que querían dar respuesta a los cuestionamientos presentados por los docentes en turno.

- El índice de reprobación disminuyó, teniéndose a final del curso por todos los grupos, un total de 10 reprobados, los cuales lograron regularizarse a tiempo.
- Las actividades permitieron que estudiantes de otros grados se interesaran en preguntar que estaban haciendo los compañeros del laboratorio de matemáticas.

Perspectivas de mejora.

Crear al menos una práctica más para el segundo semestre del ciclo escolar 2019-2020.

Instituir para el primer semestre de dicho ciclo escolar, al menos 3 prácticas de laboratorio, donde los alumnos puedan aplicar los conceptos teóricos aprendidos.

Como propuesta a futuro, está la implementación de prácticas para los otros cursos de matemáticas de la institución.

\section{Referencias}

Ceferino, L., \& Guadalupe, C. (diciembre de 2007). Las estrategias de enseñanzas lúdicas como herramienta de la calidad para el mejoramiento del rendimiento escolar y la equidad de los alumnos del nivel medio superior. REICE. Revista Iberoamericana sobre Calidad, Eficacia y Cambio en Educación, 5(5e), 60-67.

Ferreiro, R, Nuevas alternativas de aprender y enseñar, aprendizaje cooperativo, México, Trillas, 2009

García García, M. J., Fernández Sanz, L., Terrón López, M. J., Blanco Arcilla, J. "Métodos de evaluación para las competencias generales más demandada en el mercado laboral”, JENUI, 2008.

Gómez Arroyo Dánae. (2017). Propuesta de mejora a la metodología aprendizaje basado en proyectos para la enseñanza de la estadística. Puebla, BUAP.

S.A. (2012). Plan Educativo Nacional. 6 de junio de 2019, de UNAM Sitio web: http://www.planeducativonacional.unam.mx/C AP_01/Text/01_05a.html

Schmelkes Sylvia. (2001). Hacia una mejor calidad en nuestras escuelas. México: Acude 
Van Den Bergh, V., Mortermans, D., Spooren, P., Van Petegem, P., Gijbels, Dan den Bergh, V. Mortermans, D., Spooren, P., Van Petegem, P., Gijbels, D., Vanthournout, G. New assessment modes within project-based education - the stakeholders. Studies in Educational Evaluation, Kiel, Germany, v. 32, p. 345-368, 2006

Weiss, E. (2006), Diagnóstico de las prácticas y procesos curriculares en los bachilleratos integrales comunitarios, Reporte entregado a la Coordinación General de Educación Intercultural Bilingüe. 